\title{
Stochastic Analysis of Indoor THz Uplink with Co-channel Interference and Phase Noise
}

\author{
Joonas Kokkoniemi*, Alexandros-Apostolos A. Boulogeorgos ${ }^{\dagger}$, Mubarak Umar Aminu*, \\ Janne Lehtomäki*, Angeliki Alexiou ${ }^{\dagger}$, and Markku Juntti* \\ ${ }^{*}$ Centre for Wireless Communications (CWC), University of Oulu, P.O. Box 4500, 90014 Oulu, Finland \\ Email: joonas.kokkoniemi@oulu.fi \\ ${ }^{\dagger}$ Department of Digital Systems, University of Piraeus, Piraeus 18534, Greece \\ Email: al.boulogeorgos@ieee.org
}

\begin{abstract}
The paper studies the joint impact of phase noise (PN) and co-channel interference (CCI) in indoor terahertz (THz) uplink. We formulate the theoretical framework that quantifies the impact of PN on the transceiver antenna directivity by extracting exact closed-form and low-complexity tight approximations for the expected gains. Additionally, by employing stochastic geometry, we model the propagation environment of indoor $\mathbf{T H z}$ wireless systems and provide the analytical characterization of the CCI in the presence of PN, in terms of its expected value. The analysis is verified through computer simulations that reveal the accuracy of the presented theory with moderate numbers of users. The paper provides readily available tools for analyzing and designing indoor $\mathrm{THz}$ networks.
\end{abstract}

\section{INTRODUCTION}

The spectrum scarcity of the low radio frequency (RF) band has aspired the investigation of higher spectrum bands in millimeter wave (mmWave) and terahertz $(\mathrm{THz})$ regimes for the design of innovative beyond the fifth generation (B5G) wireless systems and applications [1]-[3]. The potential use cases range from short distance massive connectivity and ultra high-speed data kiosks to long distance backhaul links. Communications in these bands can offer an unprecedented increase in the bandwidth and support ultra high data rates, but they suffer from severe path attenuation [4], which can significantly limit the transmission range of the system. Moreover, the hardware imperfections of transceivers can also constrain their reliability [5].

To counterbalance the large path loss and to support larger transmission distances required in several B5G applications, such as backhauling, directional antennas have to be employed [6]-[8]. A single-input and single-output wireless communication system operating at $237.5 \mathrm{GHz}$ for transmitting data over $20 \mathrm{~m}$ at a data rate of $100 \mathrm{Gbit} / \mathrm{s}$ with antenna gains equal to $50 \mathrm{dBi}$ was reported in [6]. Furthermore, in [7], Kallfass et al. implemented an $850 \mathrm{~m}$ link at $240 \mathrm{GHz}$, in which $55 \mathrm{dBi}$ antennas were deployed. Finally, the feasibility of mmWave and $\mathrm{THz}$ high-directive wireless systems for railways was theoretically proven in [8].

From the RF design point of view, the direct conversion architecture (DCA) has received considerable attention in high frequency/data-rate systems due to its low-complexity and cost-efficient configuration [6], [9], [10]. However, DCA deployments are typically sensitive to front-end impairments, which are often inevitable due to component mismatches and manufacturing defects [11]-[13]. An indicative example is phase noise $(\mathrm{PN})$, which is caused by local oscillator (LO) imperfections and results in considerable performance degradation (see, e.g., [14] and references therein). Consequently, several published works have verified and studied the impact of PN in mmWave and $\mathrm{THz}$ wireless systems [15], [16]. For instance, in [15], Sum et al. investigated the error and throughput performance of multi-Gb/s mmWave wireless personal area networks that experience multipath fading, whereas, in [16], its impact on channel estimation and carrier recovery was highlighted.

From the network point of view, there are several studies that employ stochastic geometry to model the random node location and to extract the interference profile [17]-[27]. Most of these works assumed that the network was deployed in an infinite space. However, there are only two works that considered finite network size [26], [27]. In more detail, in [26], the authors employed stochastic geometry to model the interference levels in indoor visible light communications and, in [27], it was used for interference characterization in outdoor mmWave systems.

Despite the paramount importance of interference and PN, all the contributions in this area, including the mentioned ones, neglected their impact on the main lobe antenna gain as well as their effect on the co-channel interference (CCI), which can be caused by neighbor transceivers. Motivated by the above, this paper focuses on evaluating the joint impact of PN and $\mathrm{CCI}$ in indoor $\mathrm{THz}$ wireless systems. In this direction, we first present a model for the stochastic main antenna lobe gain reduction due to $\mathrm{PN}$, and extract an exact closed-from expression and tight low-complexity approximation for the antenna gain expected value. Building upon this model and by employing a stochastic geometry approach, we characterize the CCI in terms of its expected value. It is worth noting that stochastic geometry is a powerful mathematical tool that can be used to replace high-complexity simulations in infinite spherically or circularly symmetric networks [23]-[25]. In this work, we consider indoor scenarios, i.e., spatially limited spaces, with dimensions considerably larger than the transmission wavelength, and we verify through respective Monte Carlo simulations the accuracy of the proposed approach 


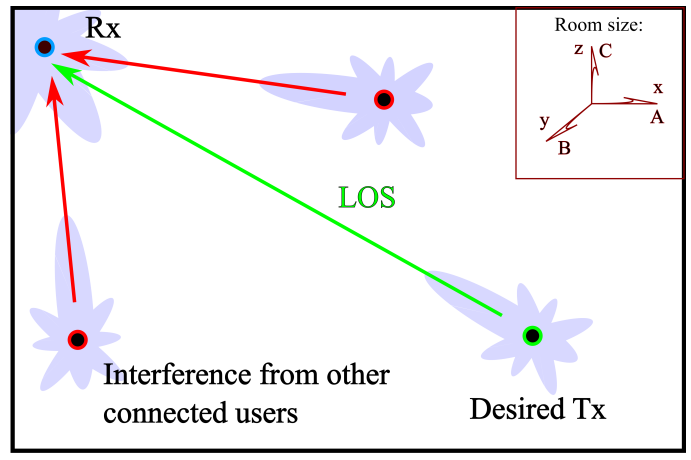

Fig. 1. The indoor system model illustration, where the $\mathrm{Rx}$ is assumed to be in the upper corner of the room in order to have a maximum visibility to the room.

and the derived expressions. Finally, our results highlight the importance of taking into account both the impact of PN and $\mathrm{CCI}$, when analyzing and designing indoor $\mathrm{THz}$ systems.

\section{SYSTEM MODEL}

As illustrated in Fig. 1, we consider an indoor uplink scenario in a $\mathrm{THz}$ network that consists of a single receiver $(\mathrm{Rx})$, i.e., the $\mathrm{THz}$ access point (TAP), and multiple transmitters (Txs). The network is assumed to be deployed within a room, which is modeled as a three dimensional rectangular space of size $A \times B \times C \mathrm{~m}^{3}$. The $\mathrm{Rx}$ resides at certain coordinates $(a, b, c)$ in the Cartesian space limited by the size of the room. The interfering Txs are randomly distributed around the room. Besides the random interfering Txs, we have a desired Tx, which determines the signal-to-interference-plus-noise ratio (SINR) levels. It is assumed that all the transmit beams of Txs are perfectly pointed towards the TAP; as a consequence, the Rx experiences interference from all the nodes. However, the receive beam of the TAP is pointed towards the desired Tx, which decreases the interference level from the random interfering Txs due to the $\mathrm{Rx}$ sees the interference mostly through the side lobes because of highly directional antennas. This makes the system uplink as in the downlink direction the $\mathrm{Rx}$ would experience interference from the side lobes of the other Tx. Thus, also the interference level is expected to be smaller due to both sides see the interference through the side lobes in contrast to the uplink where in average the $\mathrm{Rx}$ sees the Txs' main lobes through the side lobes. This case will be studied in the future work. Moreover, we assume that the ALOHA transmission scheme is employed for simplicity of analysis. The ALOHA assumption mainly contributes to the fact that the Txs are sending randomly on the same channel without any specific resource allocation.

We assume that all the transceivers are equipped with uniform linear array (ULA) antennas that consist of $N$ identical antenna elements equally spaced by distance $d$. Hence, in the absence of $\mathrm{PN}$, the complex array factor $(A F)$ can be obtained as

$$
A F(\alpha)=\beta(\Gamma) a(\alpha)=\frac{1}{\sqrt{N}} \sum_{n=0}^{N-1} e^{j \frac{2 \pi}{\lambda} d n \sin (\Gamma)} e^{j \frac{2 \pi}{\lambda} d n \sin (\alpha)}
$$

where $\beta(\Gamma)$ is the beamformer, $\Gamma$ is the beamforming direction, $a(\alpha)$ is the antenna array response, $\alpha$ is the angle of observation, $n \in\{0,1, \ldots, N-1\}$ is the antenna index, $\lambda$ is the wavelength, and $d$ is the antenna element spacing. The array power gain is then given by

$$
G(\alpha)=|A F(\alpha)|^{2} \text {. }
$$

The maximum gain of a ULA antenna is equal to the number of the antenna elements in the antenna array, i.e., $G(\Gamma)=N_{\mathrm{Tx}}$. Furthermore, since we assume linear phase array, the azimuth directivity is high and described by the above antenna array model. If we assume that the ULA antenna is formed of dipole elements, the beamwidth in elevation direction is wide. Thus, we assume herein that the antenna gain is obtained directly from the above ULA model in $3 \mathrm{D}$ environment.

It is assumed that $\mathrm{THz}$ transceivers experience $\mathrm{PN}$, which influences the $A F$ as

$$
A F_{p}(\alpha)=A F(\alpha) \gamma_{p}^{m},
$$

where $\gamma_{p}^{m}$ is the complex PN of the $m$ th RF chain and it is modeled as

$$
\gamma_{p}^{n}=\exp \left(j \theta_{k}^{m}\right),
$$

with $\theta_{k}^{m}$ being the PN angle of RF chain $m$ at time instant $k$. The phase noise is random and unique for each $\mathrm{Rx}$ chain, and, thus, $\gamma_{p}^{m}$ corrupts each RF chain $m$ independently in (1). In the numerical results, we assume that the number of RF chains is equal to the number of antenna elements. This represents a full digital beamformer. In the $\mathrm{THz}$ frequencies cheaper choice would be a hybrid structure where a single RF chain controls number of analogue phase shifters. For the sake of the analysis, full digital beamformer is considered in this paper and a comparison of the digital and hybrid beamforming is provided in the future work.

The LOs in the system can either be phased-locked or frequency-locked. When the LO is phased-locked, i.e., phasedlocked loop (PLL) is employed in the system, the PN causes a small mismatch and is normally well modeled by a Gaussian distribution. In case the system is frequency-locked, the LO in the system is tuned to the carrier frequency but it is freerunning. The PN in this case is modeled as a Wiener process [28], i.e.,

$$
\theta_{k}=\theta_{k-1}+w_{k}
$$

where $w_{k}$ is Gaussian random variable. Moreover, by assuming that the memory length of the Wiener process is $M$, the experienced PN can be expressed as

$$
\theta_{k}=\sum_{i=k-M}^{k-1} \theta_{i}+w_{k}
$$

When the phase noise is assumed to be zero mean Gaussian, this can be written as

$$
\theta_{k} \sim \mathcal{N}\left(0, M \sigma_{p}^{2}\right)
$$

due to effective sum of multiple Gaussian distributions. Note that in (7) $\sigma_{p}$ stands for the PN standard deviation. 


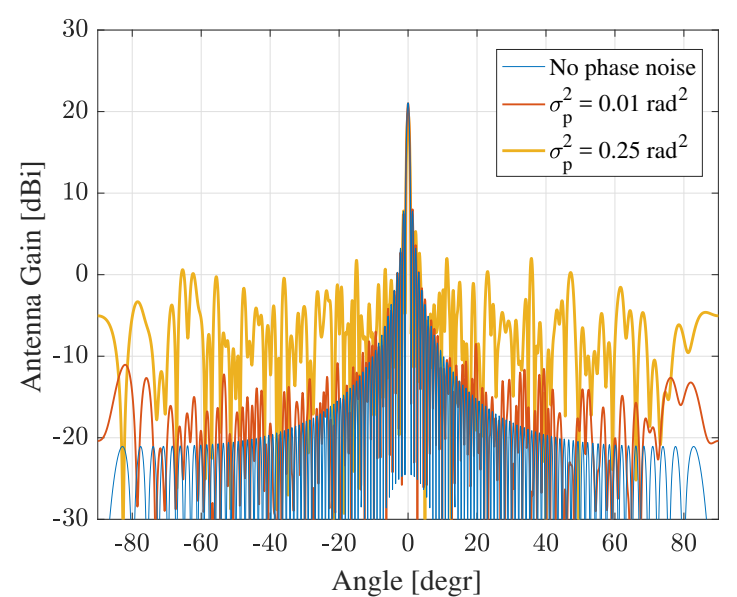

Fig. 2. Illustration of an antenna gain of the ULA model with 128 antenna elements with and and without the phase noise.

From (3), it is evident that the PN decreases the main antenna lobe gain by effectively distributing the transmitted energy to random directions and mostly to the side lobes of the antenna. This occurs through the PN modulating the beamformer $\beta(\Gamma)$ (see eq. (1)). Should the PN be absolutely random, the antenna gain would start to resemble to an omnidirectional antenna. This indicates that the distribution of $\mathrm{PN}$ is crucial to the depth of damage it does to the beamformer. The higher the PN standard deviation becomes, the more it impacts on the antenna phases. However, in realistic cases, the PN is expected to be small, but can be increased by the Wiener-type probability process with memory. An indicative example of the impact of PN in the antenna gain is provided in Fig. 2, where an antenna pattern of 128 antenna elements is considered. From this figure, we can observe that the most evident impact of the PN is on the side lobes. The impact of the phase noise on the main lobe is derived and discussed in the next section.

Finally, we utilize a line of sight (LOS) path loss model that takes into account the free space path loss (FSPL) and molecular absorption loss [29]. The LOS channel without multipath components is a valid choice in the $\mathrm{THz}$ band with high gain antennas because of the highly directional antennas with perfect beam alignment are not very efficiently radiating in NLOS directions. Similarly, the Rx does not receive much power from the NLOS directions where the Tx sends very little power in the first place. The LOS path gain of the channel can be obtained as

$$
l(r, f)=\frac{c^{2} \exp \left(-\kappa_{\mathrm{a}}(f) r\right)}{(4 \pi r f)^{2}},
$$

where $\kappa_{\mathrm{a}}(f)$ is the absorption coefficient at frequency $f, r$ is the distance from Tx to $\mathrm{Rx}$, and $c$ is the speed of light. The absorption coefficient is calculated with the help of databases [30], and as presented, e.g., in [29].

\section{EXPeCted MAIN Lobe GAIN}

The stochastic impact of the PN can be described by a mapping from the angular distribution into a unit circle. This is because the real part of the complex PN $\gamma_{p}$ describes the depth of the power degradation (or amplification). This is a consequence of the real part giving all the information of the power fluctuations due to the fact that it is directly linked to the imaginary part by the Kramers-Kronig relation. An example would be that if $\theta_{k}$ is zero, $\gamma_{p}$ is one. If $\theta_{k}$ is fully random ( 0 to $2 \pi), \gamma_{p}$ is zero mean. Therefore, we can obtain the PN impact on the main lobe gain by calculating the expected value of the real axis of unit circle by using the PDF of the PN

$$
E\left[\sqrt{G_{\mathrm{pn}}}\right]=\sqrt{N_{\mathrm{Tx}}} \int_{-\pi}^{\pi} \frac{\cos (x)}{\sqrt{2 \pi \sigma_{p}^{2}}} e^{-\frac{x^{2}}{2 \sigma_{p}^{2}}} d x,
$$

where $E\left[\sqrt{G_{\mathrm{pn}}}\right]$ is the expected amplitude antenna gain, $N_{\mathrm{tx}}$ is the number of antennas and the maximum power gain of an ideal ULA, and $\cos (x)$ maps the angles $x$ on the real axis of the unit circle. Solving this yields

$$
\begin{aligned}
& E\left[\sqrt{G_{\mathrm{pn}}}\right]= \\
& \sqrt{N_{\mathrm{Tx}}} \frac{e^{-\frac{\sigma_{p}^{2}}{2}}}{2}\left(\operatorname{erf}\left(\frac{\pi-j \sigma_{p}^{2}}{\sqrt{2} \sigma_{p}}\right)+\operatorname{erf}\left(\frac{\pi+j \sigma_{p}^{2}}{\sqrt{2} \sigma_{p}}\right)\right)
\end{aligned}
$$

where $\operatorname{erf}(x)$ is the error function. From this we can calculate that the expected antenna gain is very well approximated by

$$
E\left[G_{\mathrm{pn}}\right]=N_{\mathrm{Tx}} e^{-\sigma_{p}^{2}}
$$

for small values of $\sigma_{p}$. The phase variations need to be small enough to prevent the random phase from rotating around the unit circle for this approximation to be accurate. This approximation will be demonstrated in the numerical results to give the expected value of the antenna gain. The exact proof, alternative approximation with error, and more discussion will be addressed in future work. Other antenna patterns could be utilized as well with this model. This would be done by replacing the antenna gain term $\left(N_{\mathrm{Tx}}\right.$ herein) with the appropriate gain term describing the desired antenna pattern's main lobe gain.

\section{INTERFERENCE CHARACTERIZATION}

In this section we focus on modeling the indoor propagation environment and characterize the interference of the indoor $\mathrm{THz}$ system. The indoor propagation environment is confined by walls, which limits user distribution around the so called typical node of the network. The typical node is often assumed to be at the origin of an infinite network experiencing a similar interference as any node surrounded by random source nodes. In the derivation of the stochastic model below, we change the integration bounds to take into account the location of the typical node, or the desired Rx, in the finite network. Due to the shape of a typical room, we use Cartesian coordinate system. Note that, in the typical stochastic geometry models, the space is usually spherically symmetric about the typical node. This gives a very straightforward way to integrate the space just over the radial distance. We will show in the numerical results by simulations that the Cartesian coordinate 
based finite systems can be analysed by stochastic geometry equally accurately.

The aggregate interference at $\mathrm{Rx}$ can be analytically evaluated as [23]-[25]

$$
I_{\mathrm{aggr}}=\sum_{i \in \Phi} l\left(r_{i}\right),
$$

where

$$
l\left(r_{i}\right)=\int_{W} \frac{P_{\mathrm{Tx}}}{W} \mathbb{E}_{\Theta}\left[G_{\mathrm{Tx}}(\Theta)\right] \mathbb{E}_{\Theta}\left[G_{\mathrm{Rx}}(\Theta)\right] l\left(r_{i}, f\right) d f .
$$

with $\Phi$ being the set of interfering nodes, $P_{\mathrm{Tx}}$ representing the transmit power of the Txs, and $W$ denoting the communication bandwidth. Moreover, $\mathbb{E}_{\Theta}\left[G_{\mathrm{Tx}}(\Theta)\right]$ and $\mathbb{E}_{\Theta}\left[G_{\mathrm{Rx}}(\Theta)\right]$ are the expected antenna gains of the Txs and the $\mathrm{Rx}$, and $\Theta$ is the direction of the antenna in three dimensional space. The expected antenna gains in the context of this work are the maximum transmit powers of all Txs (desired and interference), i.e., the main lobe gains with possible phase noise impacts included. The expected antenna gain for $\mathrm{Rx}$ is the maximum gain towards the desired $\mathrm{Tx}$, and random with respect to the interfering Txs due to the fact that the $\mathrm{Rx}$ is pointed at the desired $\mathrm{Tx}$

The moments of the interference can be calculated from the Laplace transform of the aggregate interference [17], [18]

$$
\mathcal{L}_{I_{\text {aggr }}}(s)=\mathbb{E}_{\Phi}\left[\exp \left(-s \sum_{i \in \Phi} l\left(r_{i}\right)\right)\right],
$$

which can be calculated in spherical coordinate system as

$$
\mathcal{L}_{I_{\text {aggr }}}(s)=\exp \left[-2 \pi p \lambda \int_{\mathbb{R}^{3}} r(1-\exp (-s l(r))) d r\right],
$$

where $\lambda$ is the density of the nodes and $p$ is the probability of a node to transmit. When $\mathbb{R}^{3}$ in (15) is translated into the three dimensional Cartesian coordinate space, radial distance $r$ becomes

$$
r=\sqrt{x^{2}+y^{2}+z^{2}} .
$$

This allows us to confine the integration into a room sized $A \times B \times C$ for $x, y$, and $z$ axes, respectively. The $n$th raw moment of the aggregate interference can be obtained from the $n$th derivative of the Laplace transform as [20]

$$
\mathbb{E}\left[I^{n}\right]=\left.(-1)^{n} \frac{d^{n}}{d s^{n}} \mathcal{L}_{I_{\text {aggr }}}(s)\right|_{s=0},
$$

where

$$
\mathcal{L}_{I_{\text {aggr }}}(s)=\exp (-L(s)) .
$$

The term $L(s)$ in (18) is

$$
\begin{aligned}
& L(s)= \\
& 2 \pi p_{k} \lambda \int_{0-a}^{A-a} \int_{0-b}^{B-b C-c} \int_{0-c}^{C-} r(1-\exp (-s l(r))) d x d y d z .
\end{aligned}
$$

Before performing the integration, the typical node is moved to the origin in order to simplify the calculation of the distances. As a consequence, the coordinate system itself is also moved with respect to the typical node and hence the above integration bounds.

The expected interference level can be obtained as in (17) and (11) under the assumption of all the Tx antennas pointing towards the access point, i.e.

$$
\mathbb{E}_{\Theta}\left[G_{\mathrm{Tx}}(\Theta)\right]=N_{T x} e^{-\sigma_{\mathrm{Tx} / \mathrm{Rx}}^{2}} .
$$

Then, the expected interference level can be evaluated as

$$
\begin{aligned}
& \mathbb{E}\left[I_{\mathrm{aggr}}\right]=\frac{c^{2}}{8 \pi} p \lambda N_{\mathrm{Tx}} e^{-\sigma_{\mathrm{Tx}}^{2}} \\
& \times \int_{0-a}^{A-a} \int_{0-b}^{B-b} \int_{0-c}^{C-c} r^{-1} \int_{W} \frac{P_{\mathrm{Tx}}}{W f^{2}} \exp \left(-\kappa_{\mathrm{a}}(f) r\right) d f d x d y d z,
\end{aligned}
$$

where $N_{\mathrm{tx}}$ is the number of Tx elements of the interfering Txs, and $\sigma_{\mathrm{Tx}}^{2}$ is the corresponding Tx PN variance. Notice that because of the Rx being pointed at the desired $\mathrm{Tx}$, the random interfering Txs experience random $\mathrm{Rx}$ antenna gain. Therefore, the expected antenna gain is $\mathbb{E}_{\Theta}\left[G_{\mathrm{Rx}}(\Theta)\right]=1$ due to the preservation of the transmit energy and is not visible in the above equation. This behavior is validated by a simulation model where the stochastic model is utilized with unit receiver gain and the simulation model is run with actual antenna gain and random interfering Tx locations.

\section{NUMERICAL EXAMPLES}

In this section we validate the theoretical framework on PN and CCI with Monte Carlo computer simulations. The simulations were performed by dropping a Poisson distributed number of users with mean $N_{u}$ in random locations in a threedimensional rectangular volume, which is limited in $x, y$, and $z$ axes by $A, B$, and $C$, respectively. The stochastic geometry itself has been proven to be exact in the previous works [23][25]. The main purpose of the simulations is to check the validity of the antenna gain model herein. We assume that the random interfering Txs point at the $\mathrm{Rx}$ at random angles determined by their locations per simulation. The user transmit beams are all perfectly pointed at the $\mathrm{Rx}$, but the AP receive beam is pointed toward the desired Tx.

For the sake of simplicity and without loss of generality, the PN variance is assumed to be the same for all the transceivers as is the memory length of the Wiener processes. The following insightful scenario is examined. The center frequency is set to $300 \mathrm{GHz}$ and the Tx powers at equal $0 \mathrm{dBm}$ for all nodes. Morever, the number of antenna elements for the $\mathrm{Rx}$ and all the Txs is 128 , the PN variance per unit memory length is set to $0.017 \mathrm{rad}^{2}$, and the memory length of the Wiener process is assumed to vary from 1 to 80 . The Rx noise figure is $10 \mathrm{~dB}$, the communication bandwidth is $5 \mathrm{GHz}$, and the probability of transmission is $50 \%$. Furthermore, the room is assumed to be a typical small room sized $400 \times 600 \times 240$ $\mathrm{cm}^{3}(A \times B \times C)$. The desired user's Tx is at $90 \mathrm{~cm}$ away from the $\mathrm{Rx}$ (at coordinates $(x=80 \mathrm{~cm}, y=80 \mathrm{~cm}, z=150$ $\mathrm{cm})$ ), and the number of interfering users is varied from 4 


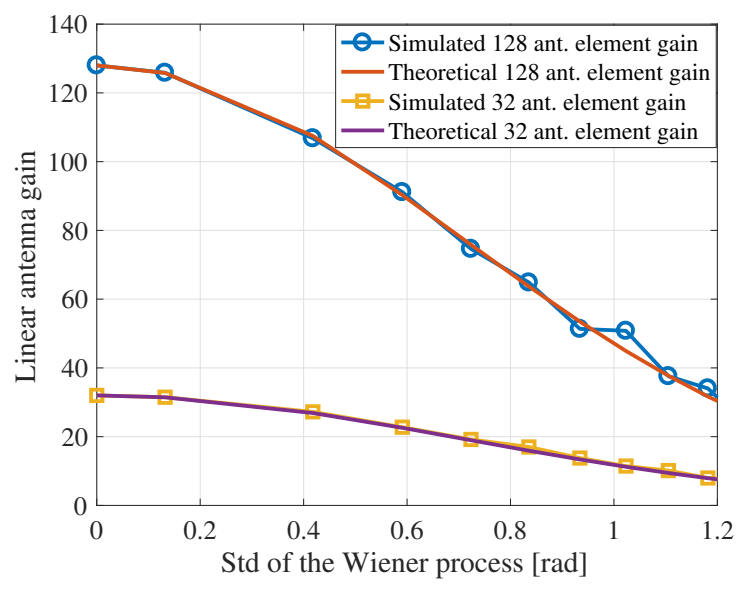

Fig. 3. Simulated and theoretical antenna gains as a function of the phase noise standard deviation.

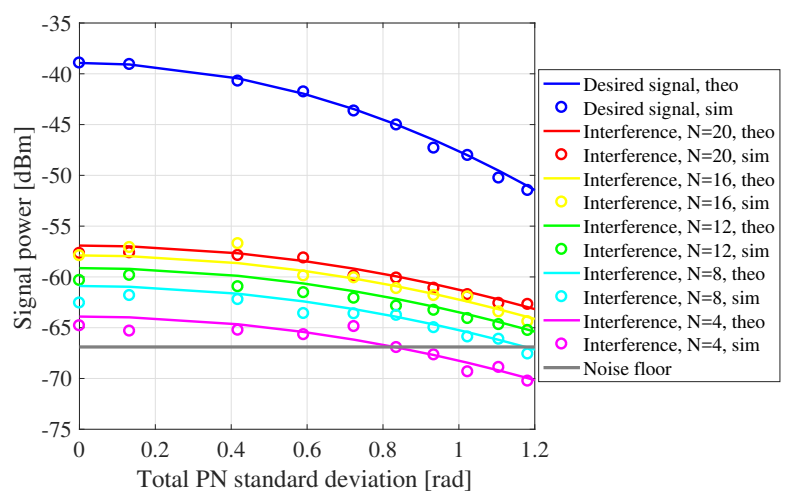

Fig. 4. Simulated and theoretical received powers for the interfering links and the desired link, as well as the noise floor as a function of the phase noise standard deviation.

to 20. The desired $\mathrm{Rx}$ is located at coordinates $(x=20 \mathrm{~cm}$, $y=20 \mathrm{~cm}, z=150 \mathrm{~cm}$ ), i.e., $20 \mathrm{~cm}$ away from the walls and at $150 \mathrm{~cm}$ height from the floor. The Monte Carlo simulations were performed over 10,000 network realizations for all the parameters.

Figure 3 shows the simulated and theoretical antenna gains as a function of the standard deviation of the PN for different numbers of antenna elements. We observe that as the PN standard deviation increases, the expected main lobe antenna gain decreases for a fixed number of antenna elements. For instance, for 128 antenna elements, as the PN standard deviation shifts from 0.2 to $0.4 \mathrm{rad}$, an approximately $12.5 \%$ antenna gain degradation occurs. Moreover, in the extreme case, in which the PN standard deviation changes from 0 to 1.2 $\mathrm{rad}$, the antenna gain degradation equals $72.7 \%$. On the other hand, for 32 antenna elements, as the PN standard deviation increases from 0.2 to $0.4 \mathrm{rad}$, an about $6.67 \%$ antenna gain reduction is observed, while, as the $\mathrm{PN}$ standard deviation shifts from 0 to $1.2 \mathrm{rad}$, the antenna gain degradation is approximately equal to $68.5 \%$. This example reveals that the same PN standard deviation shift causes a more significant antenna gain degradation as the number of antenna elements increases. We can also see that the phase noise fluctuates the

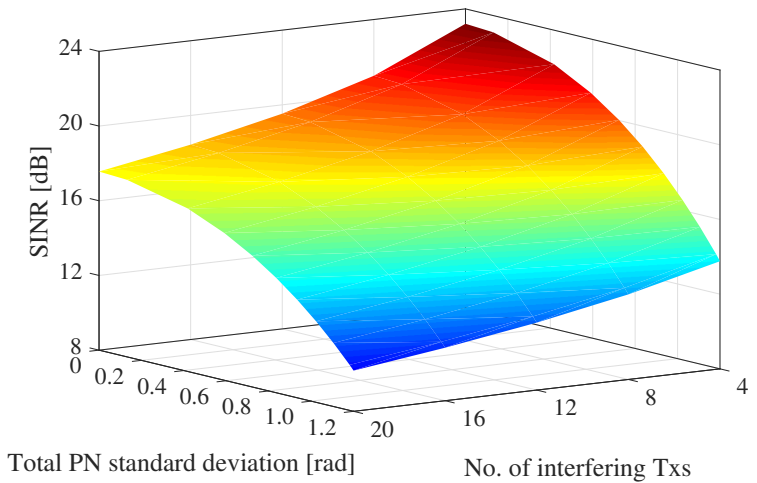

Fig. 5. Theoretical SINR as a function of the phase noise standard deviation and number of users.

antenna gain. This is best shown in the simulated antenna gains where even the averaged antenna gains fluctuate more and more as the PN variance increases. This is caused by the uncertainty the PN introduces to the designed beamformer phases.

Figure 4 illustrates the impact of the PN to the received signal powers for different values of interfering nodes. More precisely, the signal power of the desired signal accompanied by the aggregated received power of the interferers as a function of the PN standard deviation are plotted. As a benchmark, the noise floor is also depicted. Note that the markers denote the simulation results, while the continuous lines are the theoretical ones. We observe that the simulation and analytical results are identical; hence, the theoretical analysis is validated. Moreover, it is evident that for a fixed number of interfering nodes the expected antenna gains decrease as the PN standard deviation increases. Thus, the received power also decreases. The Rx has a random antenna gain with respect to the interfering Txs. This causes slightly less impact of the phase noise on the interference compared to the desired link with the main lobe gains at both ends. This has a small impact on the expected SINR as a function of the PN, which is also shown in Fig. 5 showing the stochastic SINR as a function of the PN variance and the number of the interfering users. The additional loss by PN may drive the SNR/SINR below the operational region depending on the bit error rate requirements. However, in general and for small phase noise values, the impact of the phase noise on the system performance can be considered small. The correct interpretation of the phase noise on the antenna gains is important in order to be able to model the system and its performance accurately.

\section{CONCLUSION}

This paper examined the joint impact of PN and CCI in indoor $\mathrm{THz}$ wireless systems. We provided closed-form expressions and low-complexity approximations for the expected transmit and receive antenna gain degradation due to PN. We also proved that stochastic geometry can be used for indoor confined finite $\mathrm{THz}$ networks and we capitalized this remark by extracting the analytical framework that evaluates 
the expected value of interference. Our results highlighted the importance of taking into account both the transceiver imperfections and the interference levels when analyzing and designing indoor mmWave and $\mathrm{THz}$ wireless networks.

The model herein was derived for an uplink system and we are planning to extend this work to a downlink scenario. We have also planned to update the antenna models from ULA into more representative ones used in the real mmWave and $\mathrm{THz}$ communications. In the downlink model, the desired $\mathrm{Rx}$ experiences the interference from the side lobes of the other TXs pointed at the TAP (acting as a Tx for the desired Rx). Then the impact of the phase noise is most likely larger, because the phase noise's relative gain on the side lobes is larger than that on the narrow and large gain main lobe. The main lobe, on the other hand, does experience larger power loss. That causes gain loss on the desired links, but also causes the increased side lobe interference. Understanding the sum impact of those phenomena requires dedicated analysis.

\section{ACKNOWLEDGEMENT}

This work was supported by Horizon 2020, European Union's Framework Programme for Research and Innovation, under grant agreement no. 761794 (TERRANOVA) and no. 871464 (ARIADNE). It was also supported in part by the Academy of Finland 6Genesis Flagship under grant no. 318927.

\section{REFERENCES}

[1] T. S. Rappaport et al., "Millimeter wave mobile communications for 5G cellular: It will work!" IEEE Access, vol. 1, no. 1, pp. 335-349, May 2013.

[2] A.-A. A. Boulogeorgos, A. Alexiou, T. Merkle, C. Schubert, R. Elschner, A. Katsiotis, P. Stavrianos, D. Kritharidis, P. K. Chartsias, J. Kokkoniemi, M. Juntti, J. Lehtomäki, A. Teixeirá, and F. Rodrigues, "Terahertz technologies to deliver optical network quality of experience in wireless systems beyond 5G," IEEE Commun. Mag., vol. 56, no. 6, pp. 144-151, Jun. 2018.

[3] A.-A. A. Boulogeorgos, S. Goudos, and A. Alexiou, "Users association in ultra dense THz networks," in IEEE International Workshop on Signal Processing Advances in Wireless Communications (SPAWC), Kalamata, Greece, Jun. 2018.

[4] A.-A. A. Boulogeorgos, A. Alexiou, D. Kritharidis, A. Katsiotis, G. Ntouni, J. Kokkoniemi, J. Lethtomaki, M. Juntti, D. Yankova, A. Mokhtar, J.-C. Point, J. Machodo, R. Elschner, C. Schubert, T. Merkle, R. Ferreira, F. Rodrigues, and J. Lima, "Wireless terahertz system architectures for networks beyond 5G," TERRANOVA CONSORTIUM, White paper 1.0, Jul. 2018.

[5] A.-A. A. Boulogeorgos, E. N. Papasotiriou, and A. Alexiou, "Analytical performance assessment of THz wireless systems," IEEE Access, vol. 7, no. 1, pp. 1-18, Jan. 2019.

[6] S. Koenig, D. Lopez-Diaz, J. Antes, F. Boes, R. Henneberger, A. Leuther, A. Tessmann, R. Schmogrow, D. Hillerkuss, R. Palmer, T. Zwick, C. Koos, W. Freude, O. Ambacher, J. Leuthold, and I. Kallfass, "Wireless sub-THz communication system with high data rate," Nat. Photonics, vol. 7, pp. 977 EP-, Oct. 2013.

[7] I. Kallfass, F. Boes, T. Messinger, J. Antes, A. Inam, U. Lewark, A. Tessmann, and R. Henneberger, "64 Gbit/s transmission over 850 m fixed wireless link at $240 \mathrm{GHz}$ carrier frequency," J. Infrared Milli. Terahz. Waves, vol. 36, no. 2, pp. 211-233, Feb. 2015.

[8] K. Guan, G. Li, T. Krner, A. F. Molisch, B. Peng, R. He, B. Hui, J. Kim, and Z. Zhong, "On millimeter wave and THz mobile radio channel for smart rail mobility," IEEE Trans. Veh. Technol., vol. 66, no. 7, pp. 56585674, Jul. 2017.

[9] A. J. Seeds, H. Shams, M. J. Fice, and C. C. Renaud, "Terahertz photonics for wireless communications," J. Lightwave Technol., vol. 33, no. 3, pp. 579-587, Feb. 2015.
[10] I. Kallfass, I. Dan, S. Rey, P. Harati, J. Antes, A. Tessmann, S. Wag ner, M. Kuri, R. Weber, H. Massler et al., "Towards MMIC-based $300 \mathrm{GHz}$ indoor wireless communication systems," IEICE Trans. Electron., vol. 98, no. 12, pp. 1081-1090, Dec. 2015.

[11] T. Schenk, RF Imperfections in High-Rate Wireless Systems. The Netherlands: Springer, 2008.

[12] A.-A. A. Boulogeorgos, P. C. Sofotasios, B. Selim, S. Muhaidat, G. K. Karagiannidis, and M. Valkama, "Effects of RF impairments in communications over cascaded fading channels," IEEE Trans. Veh. Technol., vol. 65, no. 11, pp. 8878 - 8894, Nov. 2016.

[13] A.-A. A. Boulogeorgos, P. C. Sofotasios, S. Muhaidat, M. Valkama, and G. K. Karagiannidis, "The effects of RF impairments in Vehicleto-Vehicle communications," in IEEE 25th International Symposium on Personal, Indoor and Mobile Radio Communications - (PIMRC): Fundamentals and PHY (IEEE PIMRC 2015 - Fundamentals and PHY), Hong Kong, P.R. China, Aug. 2015.

[14] A.-A. A. Boulogeorgos, N. D. Chatzidiamantis, and G. K. Karagiannidis, "Energy detection spectrum sensing under RF imperfections," IEEE Trans. Commun., vol. 64, no. 7, pp. 2754-2766, Jul. 2016.

[15] C. Sum, R. Funada, J. Wang, T. Baykas, M. A. Rahman, and H. Harada, "Error performance and throughput evaluation of a multigbps millimeter-wave wpan system in the presence of adjacent and cochannel interference," IEEE J. Sel. Areas Commun., vol. 27, no. 8, pp. $1433-1442$, oct 2009.

[16] R. Wang, H. Mehrpouyan, M. Tao, and Y. Hua, "Channel estimation, carrier recovery, and data detection in the presence of phase noise in ofdm relay systems," IEEE Trans. Wireless Commun., vol. 15, no. 2, pp. 1186-1205, Feb 2016.

[17] M. Haenggi and R. K. Ganti, "Interference in large wireless networks," Foundations and Trends in Networking, vol. 3, no. 2, pp. 127-248, Nov. 2008.

[18] M. Haenggi, J. G. Andrews, F. Baccelli, O. Dousse, and M. Franceschetti, "Stochastic geometry and random graphs for the analysis and design of wireless networks," IEEE J. Sel. Areas Commun., vol. 27, no. 7, pp. 1029-1046, Sep. 2009.

[19] M. Haenggi, "Outage, local throughput, and capacity of random wireless networks," IEEE Trans. Wireless Commun., vol. 8, no. 8, pp. 4350-4359, Aug. 2009.

[20] H. ElSawy, E. Hossain, and M. Haenggi, "Stochastic geometry for modeling, analysis, and design of multi-tier and cognitive cellular wireless networks: A survey," IEEE Commun. Surveys Tuts., vol. 15, no. 3, pp. 996-1019, Jun. 2013.

[21] F. Baccelli and B. Blaszczyszyn, "Stochastic geometry and wireless networks, volume I - Theory," Foundations and Trends in Networking, vol. 3, no. 3-4, pp. 249-449, Dec. 2009.

[22] A. Baddeley, Stochastic Geometry: Spatial Point Processes and their Applications, ser. Lecture Notes in Mathematics, W. Weil, Ed. Springer Berlin Heidelberg, 2007.

[23] J. Kokkoniemi, J. Lehtomäki, and M. Juntti, "Stochastic geometry analysis for mean interference power and outage probability in thz networks," IEEE Trans. Wireless Commun., vol. 16, no. 5, pp. 30173028, May 2017.

[24] - "Stochastic analysis of multi-tier nanonetworks in thz band," in Proc. ACM Int. Conf. Nanoscale Comput. Commun., 2017, pp. 1-6.

[25] _ "Stochastic geometry analysis for band-limited terahertz band communications," in IEEE Vehic. Techol. Conf. (spring), 2018, pp. 1-5.

[26] A. K. Gupta and A. Banerjee, "On the spatial performance of users in indoor vlc networks with multiple reflections," in Proc. SPCOM, 2018. [Online]. Available: https://arxiv.org/pdf/1804.04532.pdf

[27] S. M. Azimi-Abarghouyi, B. Makki, M. Nasiri-Kenari, and T. Svensson, "Stochastic geometry modeling and analysis of finite millimeter wave wireless networks," IEEE Trans. Vehic. Technol., vol. 68, no. 2, pp. 1378-1393, Feb. 2019.

[28] H. Mehrpouyan, A. A. Nasir, S. D. Blostein, T. Eriksson, G. K. Karagiannidis, and T. Svensson, "Joint estimation of channel and oscillator phase noise in mimo systems," IEEE Trans. Signal Process., vol. 60, no. 9, pp. 4790-4807, Sept. 2012.

[29] J. M. Jornet and I. F. Akyildiz, "Channel modeling and capacity analysis for electromagnetic nanonetworks in the terahertz band," IEEE Trans. Wireless Commun., vol. 10, no. 10, pp. 3211-3221, Oct. 2011.

[30] L. S. Rothman et al., "The HITRAN 2012 molecular spectroscopic database," J. Quant. Spectrosc. Radiat. Transfer, vol. 130, no. 1, pp. 4-50, Nov. 2013. 